Archive for

Organic Chemistry

Arkivoc 2018, part v, 0-0

\title{
Highly diastereoselective synthesis of rigid 3-enamino-1,5-benzodiazepines
}

\author{
Sami Chniti a , Asma Nsira a, Sadok Khouaja ${ }^{b}$, Ali Mechria a, Rafik Gharbi ${ }^{a}$, Moncef Msaddek ${ }^{\text {a }}$, \\ and Marc Lecouvey ${ }^{c *}$
}

a Laboratory of Heterocyclic Chemistry, Natural Products and Reactivity/CHPNR, Department of Chemistry, Faculty of Science of Monastir, University of Monastir, Avenue of Environment, 5019 Monastir, Tunisia ${ }^{b}$ Department of Clinical biology, Laboratory of Analysis, Treatment and valorization of Pollutants of the Environment and Products, Faculty of Pharmacy of Monastir, University of Monastir, 5019 Monastir, Tunisia

' Université Paris 13, Sorbonne Paris Cité, Laboratoire CSPBAT, CNRS UMR 7244, F-93017, Bobigny, France E-mail: marc.lecouvey@univ-paris13.fr

Received 12-08-2017

Accepted 03-01-2018

Published on line $07-03-2018$

\section{Abstract}

A variety of new (3-Z)-3-((alkyl/aryl)aminomethylene)-4-(2-hydroxyphenyl)-1,3-dihydro-2H-1,5-benzodiazepin2-ones were synthesized via addition of primary amines on the benzopyrano[4,3-c]-1H-1,5-benzodizepin-2one. High yields with excellent diastereoselectivity were obtained. The structure of cis- $\beta$-enamino- $1,5-$ benzodiazepine derivatives was characterized by 1D and 2D NMR and confirmed by an X-Ray diffraction analysis. All prepared compounds were evaluated for their in vitro antibacterial activities and promising results were given.

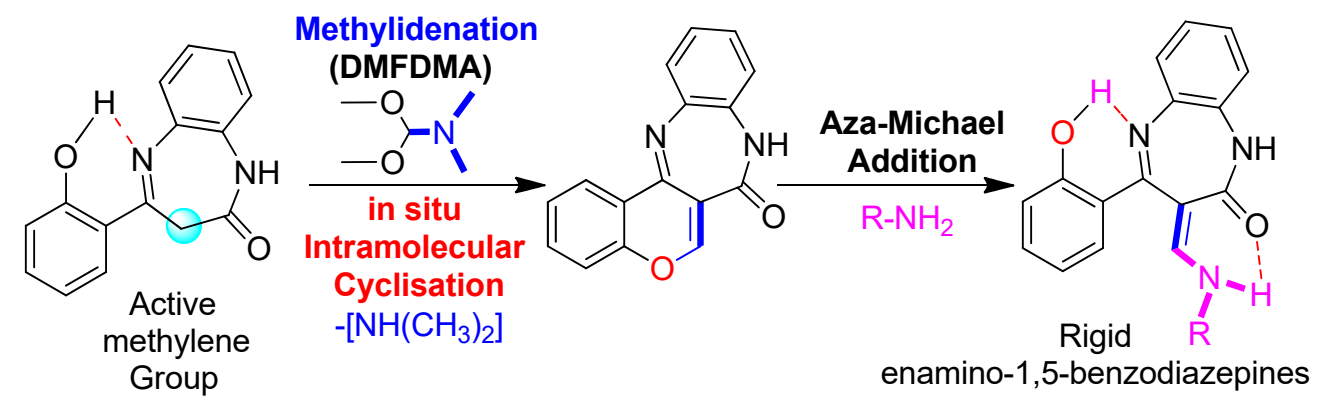

Keywords: 1, 5-Benzodiazepin-2-one, cis- $\beta$-enaminone, intramolecular hydrogen bond 


\section{Introduction}

Benzodiazepines are an important class of seven membered $N$-heterocyclic compounds reported for psychotherapeutic activities. ${ }^{1}$ Among this family, 1,5-benzodiazepine scaffolds are extremely versatile. They have been repeatedly reported to possess different clinical uses as anticonvulsant, anxiolytic and sedative agents. $^{2}$

More recently, different studies have shown new important biological properties of these compounds such as anti-HIV, anti-neuroinflammatory, ${ }^{3}$ antioxidant, ${ }^{4}$ antimicrobial, ${ }^{5}$ and antitumor, ${ }^{6}$ which have led to considerable interest in their synthesis and biological study. These biological properties are correlated with the benzodiazepine structural changes in particular by introduction of a stereogenic unit at C-3 of the benzodiazepine core. ${ }^{7}$ This modification leads to conformational restraints necessary to increase the selectivity for a particular receptor. ${ }^{8}$

In order to increase this selectivity, we are interested in the introduction of $\beta$-enaminone moiety at the $\mathrm{C3}$ position. $\beta$-Enaminones are chemical compounds which can exist in different fixed conformations owing to restricted rotations around the $\mathrm{C}=\mathrm{C}$ double bonds and the $\mathrm{C}-\mathrm{C}=\mathrm{O}$ and $\mathrm{C}-\mathrm{N}$ single bonds. ${ }^{9}$ They have synthetic utility as building blocks in preparation of many pharmaceuticals including anticonvulsant, antimalarial and antibacterial agents. ${ }^{10,11}$

The introduction of enaminone moiety at the C-3 position may reduce the flexibility of the diazepine ring, and commonly enhance its selective recognition by receptors inducing novel pharmacological activities. ${ }^{11,12}$

Herein, we propose the synthesis of novel biologically effective 1,5-benzodiazepine scaffolds from 4-(2hydroxyphenyl)-1H-1,5-benzodiazepin-2-one $1 .{ }^{13}$ To the best of our knowledge, there is only one route to access to enaminone-1,5-benzodiazepine derivatives via a transamination reaction between 3dimethylaminomethylene-1,5-benzodiazepine, and a corresponding amine. ${ }^{12,14}$

Here, we developed a new strategy from benzopyrano[4,3-c]-1,5-benzodiazepin-2-one $\mathbf{2}$ as a suitable and easily accessible starting material. In present work, the preparation of the desired derivatives and their evaluation as antibacterial and antifungal agents were described.

\section{Results and Discussion}

In our strategy, the first key intermediate was benzopyrano[4,3-c]-1,5-benzodiazepine-2-one 2 which was prepared according to literature method (scheme 1). ${ }^{15}$ Based on the recent results of the literature, the reactivity of 2 towards amines was tested.

Previously, it was shown that the $\mathrm{N}, \mathrm{N}$-bisnucleophiles are able to react on the ethylenic carbon of pyrane ring. ${ }^{16,17,18}$ We have thus studied these reaction conditions with a model amine, the $n$-butylamine in THF (Table 1). The color-change of the reaction mixture from yellow to orange was observed by the naked-eye and the progress of the reaction which was monitored by TLC showed the formation of a new only compound. After evaporation of the solvent and recrystallization in ethanol a new product was isolated albeit in low yield. On the basis of its spectral data, the isolated product, was identified as the 3-((butylamino)methylene)-4-(2hydroxyphenyl)-1H-1,5-benzodiazepin-2-one $3 \mathbf{d}$ (Table 1 ). In order to optimize the reaction conditions, we carried out the above reaction in different solvents such as: acetone, DMF and DMSO. The desired compound was isolated in good to excellent yields and we have found that DMSO was an efficient reaction medium in terms of reaction time as well as yields $(90 \%)$. 


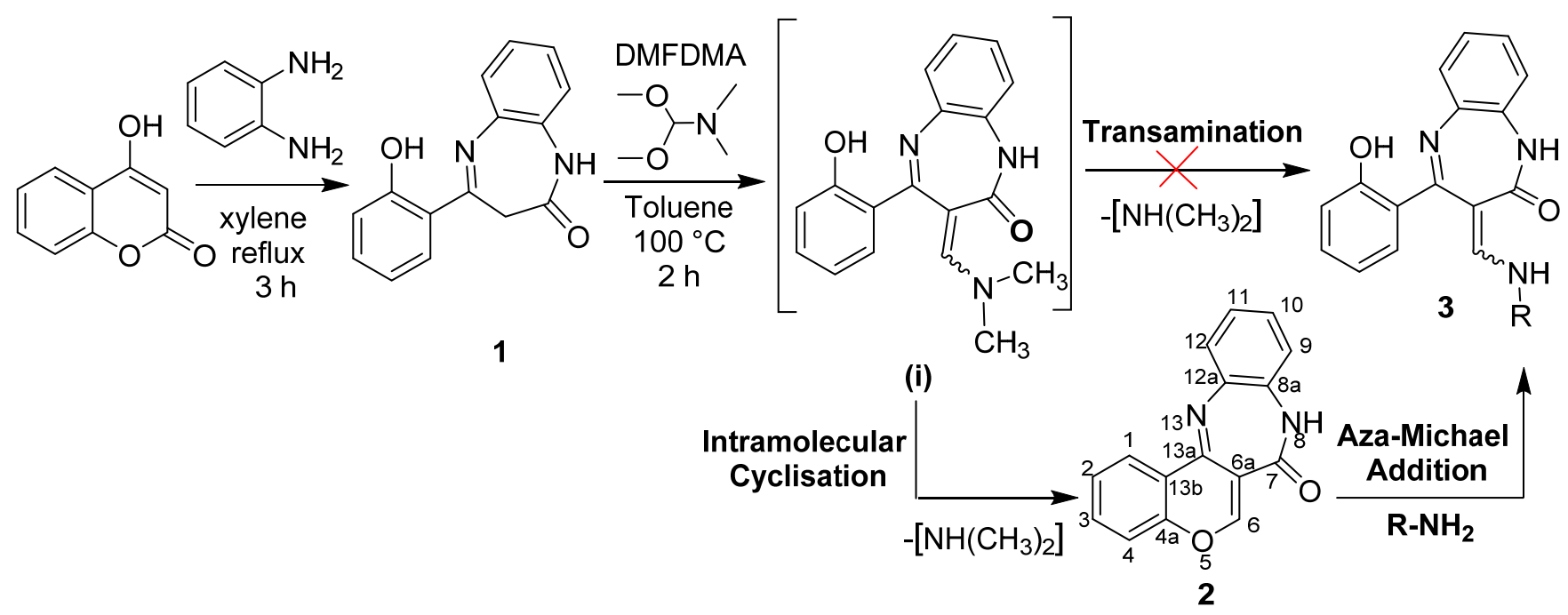

Scheme 1. Synthetic route to new 3-((alkyl/aryl)aminomethylene)-1,5-benzodiazepin-2-ones.

The ${ }^{1} \mathrm{H}$ NMR spectrum analysis of a model compound $\mathbf{3 d}$ showed characteristic signal of deshielded phenolic hydrogen singlet observed around $15 \mathrm{ppm}$. The presence of this proton confirmed a nucleophilic attack of the amino group at the C-2 of the benzopyrane moiety and the consequent ring-opening. The upfield chemical displacement indicates the presence of an intramolecular hydrogen bonding between the phenolic hydrogen and the nitrogen of the imine function. ${ }^{19}$

A careful review of the literature revealed that NMR spectroscopic techniques have been widely applied to enaminones. ${ }^{20}$ Accordingly, The configurational $\boldsymbol{E}$ - and $\boldsymbol{Z}$ - isomers can be easily distinguished by the ${ }^{1} \mathrm{H}$ NMR chemical shifts of the $\mathrm{NH}_{\text {enaminone }}$ protons: the $E$-form at high field $(\delta=4.1-6.5 \mathrm{ppm})$ and the hydrogen bonded $Z$-form at lower field $(\delta=9.5-12 \mathrm{ppm})$. The $Z$-s- $E$ conformation of enaminone-benzodiazepine $\mathbf{3 d}$ can be clearly deduced from the set of both doublet $(6.91 \mathrm{ppm}, 1 \mathrm{H})$ and the broadened multiplet $(8.80 \mathrm{ppm}, 1 \mathrm{H})$ assigned to $\mathrm{H}-1^{\prime}$ and $\mathrm{H}-2^{\prime}$ ( $\mathrm{NH}_{\text {enaminone) }}$ respectively, due to restricted rotation around the $\mathrm{C}-\mathrm{N}$ single bond (Table 1).

Additionally, single crystals suitable for an X-Ray diffraction study were grown for $\mathbf{3} \mathbf{d}$ by crystallization in $\mathrm{CH}_{2} \mathrm{Cl}_{2} / n$-Hexane. The crystal structure of the enaminone-1,5-benzodiazepines $\mathbf{3 d}$ shown in Figure 5 allowed to establish that the solid state structure contains only the $\mathbf{Z}$-isomer found in solution.

As it can be observed from the ORTEP view of derivative $\mathbf{3} \mathbf{d}$, the benzodiazepine skeleton is not planar with the folding of the seven membered ring which adopts a boat-like conformation. ${ }^{21}$ It is important to note also, that the molecular structure reveals two strong intramolecular hydrogen bonds. The first one, between the phenolic hydrogen and the nitrogen $\mathrm{N}$ of the imine function [ $\mathrm{d}(\mathrm{O} 1-\mathrm{H} 1 \cdots \mathrm{N} 2)$ of $1.81 \AA$ ] and the second one is along the heterodienic $\mathrm{O}=\mathrm{C}-\mathrm{C}=\mathrm{C}-\mathrm{NH}$ moiety, which adopts the form of six membered ring [d (N3-H3 ‥O2) of $2.12 \AA$ ] conferring to the structure more rigidity. The dihedral angle of $169.76^{\circ}$, between the protons in the $=\mathrm{CH}-\mathrm{NH}$ moiety, is in accordance with the one estimated by ${ }^{1} \mathrm{H}$ NMR in solution using the coupling constant and the Karplus type equation. ${ }^{22}$

In the light of these findings, the proposed reaction mechanism is a simple Michael/retro-Michael sequence involving primary amines. The starting material $\mathbf{2}$ has the same reactivity as an $\alpha, \beta$-unsaturated ketone as well as an $\alpha, \beta$-unsaturated imine system. This compound is able to undergo two different nucleophilic attacks of amines on the most electrophilic position (carbon C6) (pathway I or pathway II, Scheme 2 ). In the initial step, 1,4-conjugate addition (aza-Michael addition) can lead to $\beta$-aminoenol intermediate $\left(\mathbf{i}_{1}\right)$ or to $\beta$-amino enamine intermediate $\left(\mathbf{i}_{2}\right)$. 
Table 1. Study of the reaction conditions.

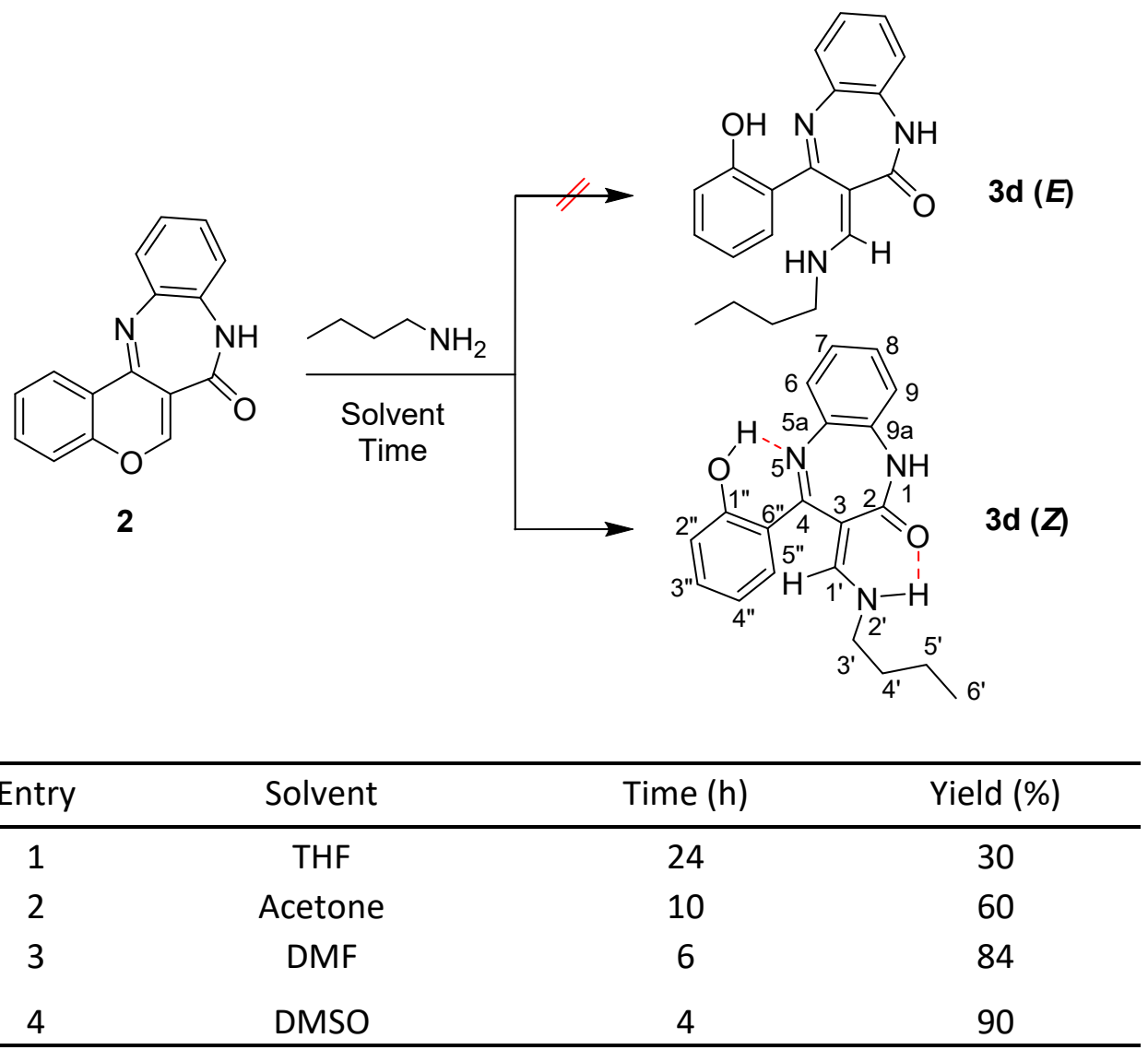

As a second step, these two potential intermediates can be converted, respectively by enol-ketone and enamine-imine tautomerisms, into an unstable intermediate $\left(\mathbf{i}_{3}\right)$. A retro-oxa-Michael addition, with a subsequent carbon-oxygen bond cleavage of the benzopyrane moiety, gives the desired compound stabilized by intramolecular hydrogen bonding.

To study the efficiency of this method various substituted amines were used. In all cases, the desired new compounds 3a-m were obtained in yields ranging from $50 \%$ to $90 \%$ (Table 2 ).

Aliphatic amines such as ethylamine, isopropylamine, $n$-butylamine and isobutylamine reacted with $\mathbf{2}$ in DMSO giving the desired products in good yields. However, the reaction was sluggish with the substrates: tertbutylamine 2,4,4-trimethylpentan-2-amine and sec-butylamine, this may be due to the steric hindrance of methyl groups in the proximity of the amino group. In aromatic series, the reaction is less efficient and reaction times are longer because of the weak nucleophilicity of the amine.

The structure of the prepared compounds 3a-m was completely characterized by NMR. High Resolution Mass Spectrometry (HRMS) data of all the formed derivatives were also in agreement with the proposed structures. 


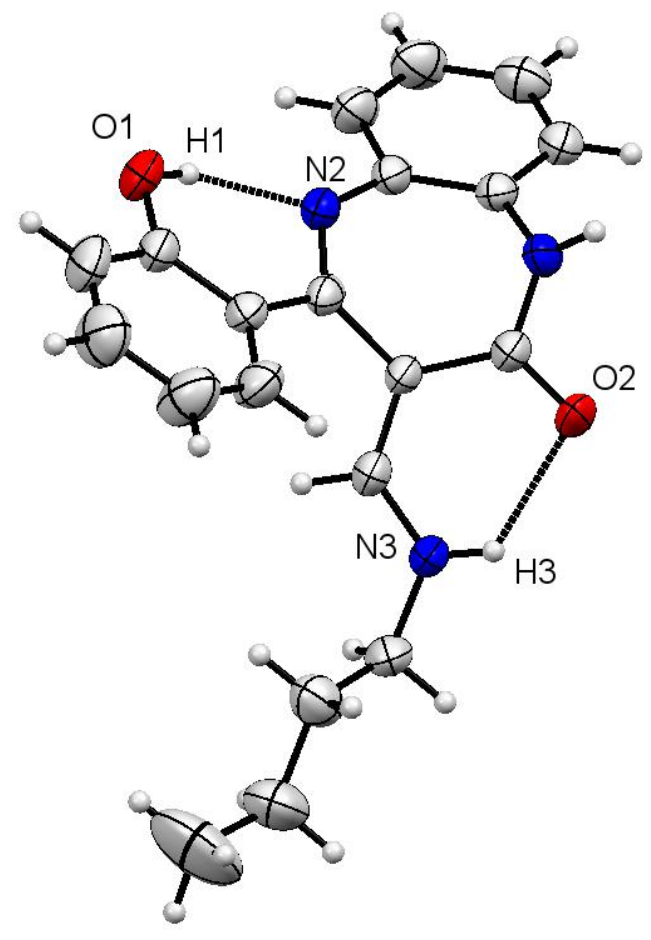

Figure 5. ORTEP representation of the molecular structure of $\mathbf{3 d}$ with atom labeling scheme and anisotropic displacement ellipsoid depicted at $50 \%$ probability $\left(293^{\circ} \mathrm{K}\right)$.

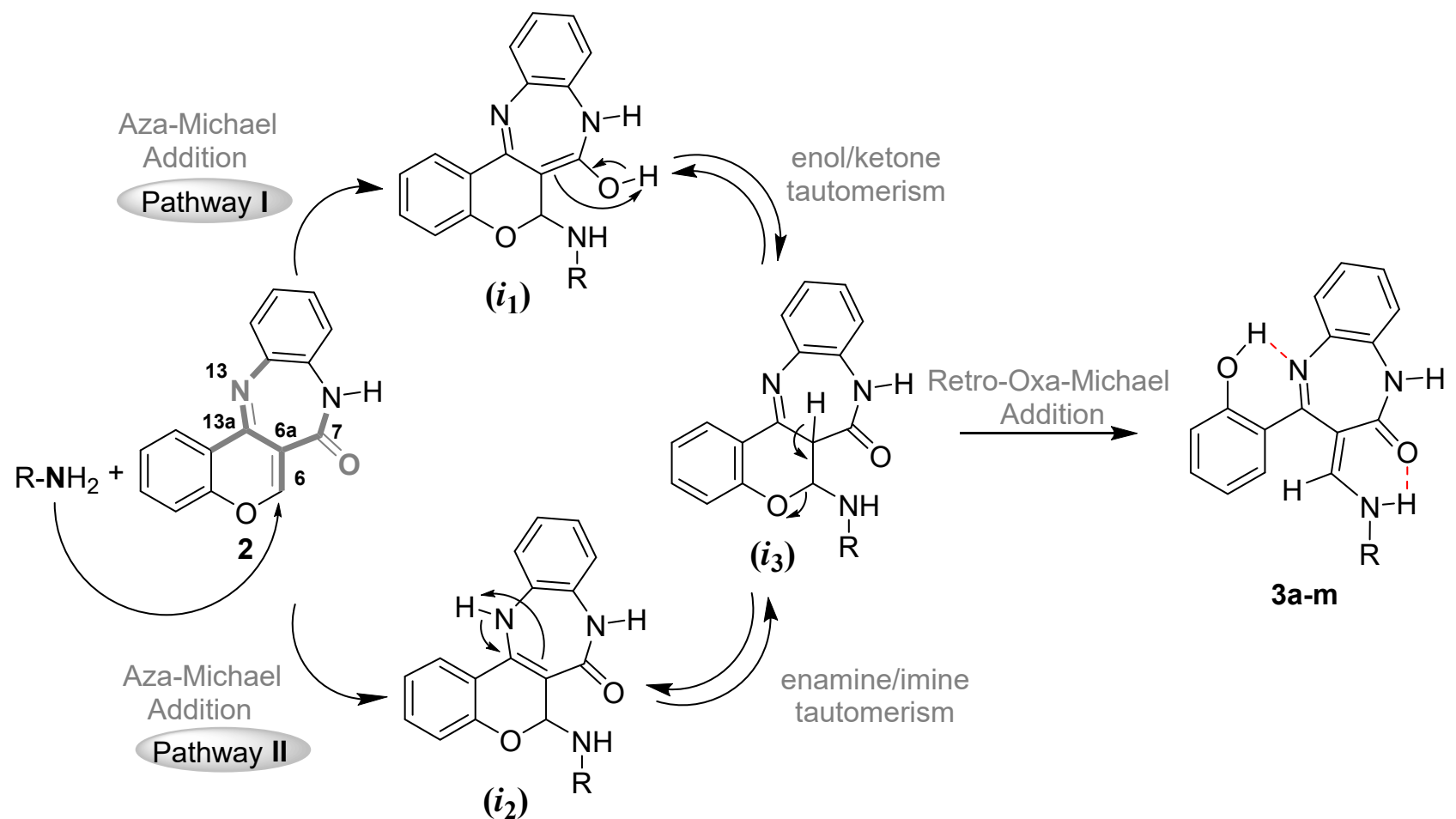

Scheme 2. Proposed mechanism of enaminone-1,5-benzodiazepines 3a-m formation. 
The ${ }^{1} \mathrm{H}$ NMR and ${ }^{13} \mathrm{C}$ NMR data of 3a-m confirmed the existence of the same conformer for each compound. In fact, the ${ }^{1} \mathrm{H}$ NMR spectrum showed only one doublet between $6.5 \mathrm{ppm}$ and $7.5 \mathrm{ppm}$

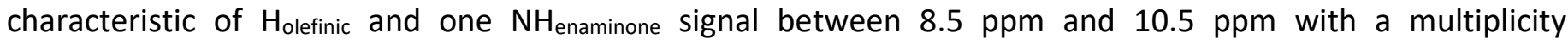
depending on the amine structure (Table 3).

For all enaminones $3 \mathrm{a}-\mathrm{m}$, the chemical shift of the $\mathrm{NH}_{\text {enaminone }}$ proton was found at lower field due to the deshielded effect of the $\mathrm{C}=\mathrm{O} \cdots \mathrm{HN}$ type of intramolecular hydrogen bonding. These spectral data proved that the reaction led exclusively to the $\boldsymbol{Z}$-form. Furthermore, the vicinal coupling constants ${ }^{3} \mathrm{~J}=\mathrm{CH}-\mathrm{NH}$-naminone $(12.1$ $14.1 \mathrm{~Hz}$ ) for the $=\mathrm{CH}-\mathrm{NH}_{\text {enaminone }}$ moiety of each derivative indicated a dihedral angle of $180^{\circ}$ which was in agreement with the s-trans conformation. We concluded also that the reaction was conducted with complete diastereoselectivity for both aromatic and aliphatic amines.

Table 2. Preparation of cis- $\beta$-enaminone-1,5-benzodiazepines 3a-m in DMSO

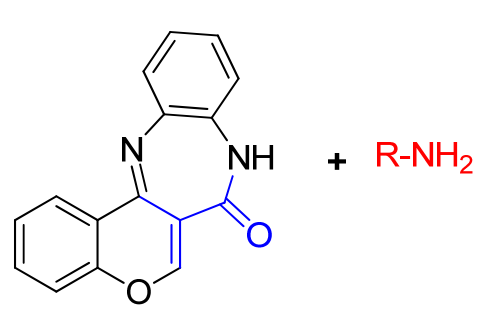

2

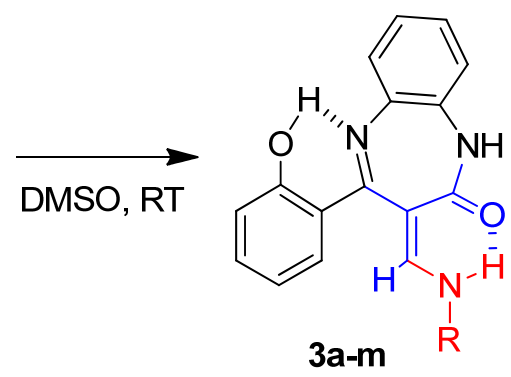

\begin{tabular}{|c|c|c|c|c|}
\hline Entry & $\mathrm{R}$ & Compound & Time (h) & $\begin{array}{c}\text { Yields } \\
(\%)^{a}\end{array}$ \\
\hline 1 & $\mathrm{Me}$ & $3 a$ & 3 & 52 \\
\hline 2 & Et & $3 b$ & 2 & 78 \\
\hline 3 & $\mathrm{iPr}$ & $3 c$ & 2 & 85 \\
\hline 4 & $n-\mathrm{Bu}$ & $3 d$ & 4 & 90 \\
\hline 5 & $\begin{array}{l}2,4,4- \\
\text { trimethylpentan-2-yl }\end{array}$ & $3 e$ & 3 & 80 \\
\hline 6 & tert-Bu & $3 f$ & 3 & 77 \\
\hline 7 & $\mathrm{iBu}$ & $3 g$ & 3 & 85 \\
\hline 8 & $s e c-B u$ & $3 h$ & 3 & 85 \\
\hline 9 & $n$-Hex & $3 \mathbf{i}$ & 5 & 65 \\
\hline 10 & Cyhex & $3 \mathbf{j}$ & 5 & 84 \\
\hline 11 & $\mathrm{C}_{6} \mathrm{H}_{5}$ & $3 k$ & 12 & 55 \\
\hline 12 & $4-\mathrm{MeOC}_{6} \mathrm{H}_{4}$ & 31 & 10 & 60 \\
\hline 13 & $4-\mathrm{ClC}_{6} \mathrm{H}_{4}$ & $3 m$ & 12 & 50 \\
\hline
\end{tabular}

${ }^{\text {a } I s o l a t e d ~ Y i e l d ~}$ 
Table 3. ${ }^{1} \mathrm{H}$ NMR data for 3a-m

\begin{tabular}{|c|c|c|c|c|}
\hline \multirow[t]{2}{*}{ Compounds } & \multicolumn{2}{|c|}{$\mathrm{H}^{\mathrm{a}}$ olefinic } & \multicolumn{2}{|c|}{$\mathrm{H}-\mathrm{N}^{\mathrm{b}}$ enaminone } \\
\hline & $\delta(\mathrm{ppm})$ & ${ }^{3} \mathrm{~J}=\mathrm{CH}-\mathrm{NHenaminone}(\mathrm{Hz})$ & $\delta$ (ppm) & ${ }^{3} \mathrm{~J}=\mathrm{CH}-\mathrm{NHenaminone}(\mathrm{Hz})$ \\
\hline $3 a$ & $6.88(d)$ & 13.8 & $8.56-8.73(\mathrm{~m})$ & / \\
\hline $3 b$ & $6.93(d)$ & 13.8 & $8.68-8.78(\mathrm{~m})$ & / \\
\hline $3 c$ & $6.98(d)$ & 14.1 & $8.69-8.76(\mathrm{~m})$ & / \\
\hline 3d & $6.91(d)$ & 13.8 & $8.64-8.76(\mathrm{~m})$ & / \\
\hline $3 e$ & $7.05(d)$ & 14.1 & $9.27(d)$ & $14.1 \mathrm{~Hz}$ \\
\hline $3 f$ & $7.06(d)$ & 14.4 & 9.05 (d) & $14.4 \mathrm{~Hz}$ \\
\hline $3 g$ & $6.89(d)$ & 13.8 & $8.38-8.88(m)$ & / \\
\hline $3 \mathrm{~h}$ & $6.96(d)$ & 13.8 & $8.7-8.81(m)$ & / \\
\hline $3 \mathbf{i}$ & $6.93(d)$ & 13.8 & $8.71-8.90(\mathrm{~m})$ & / \\
\hline $3 \mathbf{j}$ & $6.99(d)$ & 13.8 & $8.82(d)$ & 13.8 \\
\hline $3 k$ & $7.34(d)$ & 13.2 & $10.17(d)$ & 13.2 \\
\hline 31 & $7.20(d)$ & 12.0 & $10.24(d)$ & 12.0 \\
\hline $3 m$ & $6.90(d)$ & 12.1 & $10.20(d)$ & 12.1 \\
\hline
\end{tabular}

All of the benzodiazepines derivatives 3a-m were tested for antibacterial activities against both standard Gram-positive and Gram-negative bacterial strains. Their minimum inhibitory concentrations (MICs $\mu \mathrm{g} / \mathrm{ml}$ ) are shown in Table 4. Streptomycin was used as reference compound. It has been observed that some of the compounds exhibited interesting antibacterial activity against both Gram-positive and Gram-negative bacteria (Table 4) showing moderate to excellent activities against the used microorganisms. Indeed, compounds 3a-c, 3f-i and $\mathbf{3 m}$ showed effective activity against M. luteus (Table 4, entries 1-3; 6-9 and 13, MIC=0.125-0.5 $\mu \mathrm{g} / \mathrm{mL}$ ) compared to that of Streptomycin $(\mathrm{MIC}=2 \mu \mathrm{g} / \mathrm{mL}$ ). Moreover, compounds 3f-k and 3m showed effective activity against E.feacalis (Table 4, entries 6-11 and $13, \mathrm{MIC}=0.125-0.5 \mu \mathrm{g} / \mathrm{mL}$ ) compared to that of antibacterial standard ( $\mathrm{MIC}=0.5 \mu \mathrm{g} / \mathrm{mL}$ ). The obtained data revealed also, that compounds $\mathbf{3 e - f}$ and $\mathbf{3} \mathbf{j}-\mathbf{k}$ might be the major active compounds against $E$. coli and exhibited a similar activity (Table 4, entries 5-6; 10-11 , $\mathrm{MIC}=0.25 \mu \mathrm{g} / \mathrm{mL}$ ). Compound $3 \mathrm{~m}$ was significantly the most potent derivative against all used bacteria (Table 4, entry $13, \mathrm{MIC}=0.125-2 \mu \mathrm{g} / \mathrm{mL}$ ). This effect may be explained by the importance of the 4chlorophenylamino system for the antibacterial activity of the enaminone-benzodiazepinic analogs. Most of the tested compounds displayed poor activity against $P$. aeruginosa known by its resistance against many antibiotic agents. This was very clear from the higher amounts of these compounds (MIC $=4-8 \mu \mathrm{g} / \mathrm{mL}$ ) that were required for the inhibition of this bacteria.

Table 4. Antibacterial activity of compounds 3a-m, expressed in MIC $(\mu \mathrm{g} / \mathrm{mL})$

\begin{tabular}{|c|c|c|c|c|c|c|c|c|c|c|}
\hline \multirow{2}{*}{\multicolumn{2}{|c|}{$\begin{array}{l}\text { Entry and } \\
\text { Compound }\end{array}$}} & \multicolumn{5}{|c|}{ Gram-positive bacteria } & \multicolumn{4}{|c|}{ Gram-negative bacteria } \\
\hline & & \multirow{2}{*}{$\frac{\text { M.L. }^{1}}{0.25}$} & \multirow{2}{*}{$\frac{\text { B.C. }^{2}}{1}$} & \multirow{2}{*}{$\frac{\text { S.A. }{ }^{3}}{1}$} & \multirow{2}{*}{$\frac{\text { S.E. }^{4}}{0.5}$} & \multirow{2}{*}{$\frac{\text { E.F. }^{5}}{0.5}$} & \multirow{2}{*}{$\frac{\text { E.C. }{ }^{6}}{2}$} & \multirow{2}{*}{$\frac{\text { P.A. }{ }^{7}}{8}$} & \multirow{2}{*}{$\frac{\text { S.T. }{ }^{8}}{4}$} & \multirow{2}{*}{$\frac{\text { L. }^{9}}{8}$} \\
\hline 1 & $3 a$ & & & & & & & & & \\
\hline 2 & $3 b$ & 0.25 & 1 & 1 & 1 & 1 & 2 & 8 & 2 & 4 \\
\hline 3 & $3 c$ & 0.5 & 0.25 & 0.25 & 0.5 & 0.5 & 1 & 8 & 0.25 & 0.5 \\
\hline 4 & $3 d$ & 2 & 1 & 0.25 & 1 & 8 & 8 & 2 & 4 & 4 \\
\hline
\end{tabular}


Table 4 (continued)

\begin{tabular}{|c|c|c|c|c|c|c|c|c|c|c|}
\hline \multirow{2}{*}{\multicolumn{2}{|c|}{$\begin{array}{l}\text { Entry and } \\
\text { Compound }\end{array}$}} & \multicolumn{5}{|c|}{ Gram-positive bacteria } & \multicolumn{4}{|c|}{ Gram-negative bacteria } \\
\hline & & \multirow{2}{*}{$\frac{\text { M.L. }^{1}}{2}$} & \multirow{2}{*}{$\frac{\text { B.C. }^{2}}{0.25}$} & \multirow{2}{*}{$\frac{\text { S.A. }}{1}$} & \multirow{2}{*}{$\frac{\text { S.E. }{ }^{4}}{2}$} & \multirow{2}{*}{$\frac{\text { E.F. }}{8}$} & \multirow{2}{*}{$\begin{array}{l}\text { E.C. }{ }^{6} \\
0.25\end{array}$} & \multirow{2}{*}{$\frac{\text { P.A. }{ }^{7}}{2}$} & \multirow{2}{*}{$\frac{\text { S.T. }}{4}$} & \multirow{2}{*}{$\frac{\text { L.M }^{9}}{0.5}$} \\
\hline 5 & $3 e$ & & & & & & & & & \\
\hline 6 & $3 f$ & 0.125 & 2 & 2 & 0.5 & 0.125 & 0.25 & 8 & 4 & 4 \\
\hline 7 & $3 g$ & 0.25 & 2 & 2 & 0.5 & 0.25 & 0.5 & 4 & 4 & 4 \\
\hline 8 & $3 \mathrm{~h}$ & 0.125 & 1 & 2 & 0.5 & 0.25 & 0.5 & 8 & 4 & 8 \\
\hline 9 & $3 \mathbf{i}$ & 0.5 & 1 & 1 & 2 & 0.25 & 2 & 8 & 2 & 2 \\
\hline 10 & $3 \mathbf{j}$ & 2 & 4 & 1 & 2 & 0.25 & 0.25 & 2 & 4 & 1 \\
\hline 11 & $3 k$ & 2 & 0.5 & 1 & 2 & 0.25 & 0.25 & 8 & 0.25 & 4 \\
\hline 12 & 31 & 2 & 1 & 1 & 2 & 1 & 0.5 & 4 & 4 & 8 \\
\hline 13 & $3 m$ & 0.5 & 0.125 & 0.5 & 0.5 & 0.125 & 0.125 & 2 & 0.25 & 1 \\
\hline \multicolumn{2}{|c|}{ Streptomycin } & 2 & 0.5 & 2 & 0.5 & 0.5 & 0.5 & 2 & 0.5 & 2 \\
\hline
\end{tabular}

${ }^{1}$ Micrococcus luteus; ${ }^{2}$ Bacillus cereus; ${ }^{3}$ Staphylococcus aureus; ${ }^{4}$ Staphylococcus epidermidis ; ${ }^{5}$ Enterococcus faecalis ; ${ }^{6}$ Escherichia coli $;{ }^{7}$ Pseudomonas aeruginosa ; ${ }^{8}$ Salmonella typhimurium ; ${ }^{9}$ Listeria monocytogenes ; $\mathrm{MIC}(\mu \mathrm{g} / \mathrm{mL})$, minimum inhibitory concentration, i.e., the lowest concentration of the compound to inhibit the growth of bacteria completely.

\section{Conclusions}

In summary, we have disclosed an efficient access to new (3Z)-3-((alkyl/aryl)aminomethylene)-4-(2hydroxyphenyl)-1,3-dihydro-2H-1-benzodiazepin-2-ones. Simple workup, easy isolation, good yields and high diastereoselectivity for the desired products under mild conditions are the best features of the present methodology. These novel cis- $\beta$-enaminone-1,5-benzodiazepines present a new class of benzodiazepine derivatives which are promising systems to exhibit new pharmacological activities. Their evaluation as antimicrobial agents showed that most of the compounds exhibited moderate to good action on bacterial species.

\section{Experimental section}

General. Reagents were prepared in laboratory or were commercial products of analytical purity (Merck, Fluka, and Aldrich) and used as received. The Mueller-Hinton agar which was used in antibacterial susceptibility testing, was purchased from Merck. Melting points were taken on a Buchi-510 capillary apparatus and are uncorrected. ${ }^{1} \mathrm{H},{ }^{13} \mathrm{C}$ and two-dimensional NMR spectra were recorded with $\mathrm{AC}-300$ Bruker spectrometer at room temperature (rt) in $\mathrm{CDCl}_{3}$ at $300 \mathrm{MHz}$ and at $75 \mathrm{MHz}$, using residual non deuterated solvent peaks as internal reference. Coupling constants are given in $\mathrm{Hz}$. IR spectra were recorded on a Perkine Elmer Spectrum two FT-IR instrument with the Universal ATR Sampling Accessory. HRMS spectra were acquired with an electrospray- time-of-flight (ESI-TOF, LCT Premier XE, Waters) mass spectrometer in the positive ion mode. All reactions were followed by TLC using aluminium sheets of Merck silica gel $60 F_{254}, 0.2$ $\mathrm{mm}$. The starting materials $\mathbf{1}$ and $\mathbf{2}$ were prepared according to the literature. ${ }^{13,15}$

\section{General procedure for the synthesis of the enaminone-1,5-benzodiazepinones 3(a-m).}


To a stirred suspension of compound $2(262 \mathrm{mg}, 1 \mathrm{mmol})$ in DMSO $(10 \mathrm{~mL})$ was added the aliphatic amines (1,5 eq). The solution was allowed to react for 2 to 12 hours and its progress was monitored by a TLC control (cyclohexane-ethyl acetate 80:20). After the completion of the reaction, the reaction mixture was extracted with dichloromethane $(15 \mathrm{~mL})$. The organic layer was dried over $\mathrm{MgSO}_{4}$ and solvents were removed in vacuum. Diethyl ether was added to the resulted yellow crude oil, the so-formed yellow precipitate was collected and recrystallized from ethanol to afford the corresponding pure 3a-m in $50-90 \%$ yields.

(3Z)-4-(2-hydroxyphenyl)-3-((methylamino)methylene)-1,3-dihydro-2H-1,5-benzodiazepin-2-one (3a)

Yellow solid; yield 52\%, mp 180-183 ${ }^{\circ} \mathrm{C} ;{ }^{1} \mathrm{H}$ NMR $\left(300 \mathrm{MHz}, \mathrm{CDCl}_{3}\right) \delta_{\mathrm{H}}: 3.01\left(\mathrm{~d}, 3 \mathrm{H}, \mathrm{CH}_{3}, J 5.1 \mathrm{~Hz}\right), 6.47(\mathrm{~s}, 1 \mathrm{H}$, $\left.\mathrm{NH}_{\text {bzd }}\right)$, 6.77-6.83 (m, 2Harom), $6.88\left(\mathrm{~d}, 1 \mathrm{H}, \mathrm{H}_{\text {olefinic, }} J 13.8 \mathrm{~Hz}\right)$ 6.99-7.46 (m, 6 $\left.\mathrm{H}_{\text {arom }}\right), 8.56-8.73(\mathrm{~m}, 1 \mathrm{H}$, NHenaminone), 15.12 (bd-s, $1 \mathrm{H}, \mathrm{OH}) .{ }^{13} \mathrm{C} N M R\left(75.47 \mathrm{MHz}, \mathrm{CDCl}_{3}\right) \delta_{\mathrm{c}}: 29.7,95.1,117.7,118.2,120.7,121.5$, 124.6, 126.7, 127.6, 130.8, 132.7, 132.9, 137.0, 157.8, 163.2, 167.6, 178.3; IR (ATR) $v_{\max } / \mathrm{cm}^{-1}: 3280-3170(\mathrm{NH})$, $3027(\mathrm{OH}) 1640$ (C=O), 1577 (C=N); HRMS (ESI-TOF) for $\mathrm{C}_{17} \mathrm{H}_{15} \mathrm{~N}_{3} \mathrm{O}_{2}[\mathrm{M}+\mathrm{H}]+$ : calcd, 294.1164 found 294.1155.

(3Z)-3-((ethylamino)methylene)-4-(2-hydroxyphenyl)-1,3-dihydro-2H-1,5-benzodiazepin-2-one (3b) Yellow solid, yield 78\%; mp: 188-191 ${ }^{\circ} \mathrm{C}$; ${ }^{1} \mathrm{H}$ NMR $\left(300 \mathrm{MHz}, \mathrm{CDCl}_{3}\right) \delta_{\mathrm{H}}: 1.22\left(\mathrm{t}, 3 \mathrm{H}, \mathrm{CH}_{3}, J 7.2 \mathrm{~Hz}\right.$ ), 3.25 (qt, $2 \mathrm{H}, \mathrm{CH}_{2},{ }^{3} \mathrm{~J}_{1}$ $\left.7.2 \mathrm{~Hz},{ }^{3} J_{2} 6.6 \mathrm{~Hz}\right), 6.49(\mathrm{~s}, 1 \mathrm{H}, \mathrm{NH}$ bzd $), 6.79-6.86\left(\mathrm{~m}, 2 \mathrm{H}_{\text {arom }}\right), 6.93\left(\mathrm{~d}, 1 \mathrm{H}, \mathrm{H}_{\text {olefinic }} J 13.8 \mathrm{~Hz}\right), 6.99-7.44(\mathrm{~m}$, $\left.6 \mathrm{H}_{\text {arom }}\right), 8.67(\mathrm{~m}, 1 \mathrm{H}, \mathrm{NH}$ enaminone), 15.12 (bd-s, $1 \mathrm{H}, \mathrm{OH}) .{ }^{13} \mathrm{C} \mathrm{NMR}\left(75.47 \mathrm{MHz}, \mathrm{CDCl}_{3}\right) \delta_{\mathrm{c}}: 16.2,43.9,95.2,117.6$, 118.2, 120.7, 121.6, 124.5, 126.8, 127.7, 130.8, 131.9, 133.5, 137.0, 157.5, 163.2, 168.0, 178.3; IR (ATR) $v_{\max } / \mathrm{cm}^{-1}$ : $3287-3174(\mathrm{NH}), 3047(\mathrm{OH}) 1644(\mathrm{C}=\mathrm{O}), 1576(\mathrm{C}=\mathrm{N})$; HRMS (ESI-TOF) for $\mathrm{C}_{18} \mathrm{H}_{18} \mathrm{~N}_{3} \mathrm{O}_{2}$ [M + H]+: calcd 308.1321 found 307.1315 .

(3Z)-4-(2-hydroxyphenyl)-3-((isopropylamino)methylene)-1,3-dihydro-2H-1,5-benzodiazepin-2-one (3c)

Yellow solid, yield 85\%, mp: $150-153{ }^{\circ} \mathrm{C} ;{ }^{1} \mathrm{H}$ NMR $\left(300 \mathrm{MHz}^{\mathrm{C} C D C l}\right) \delta_{\mathrm{H}}: 1.24\left(\mathrm{~d}, 6 \mathrm{H}, 2 \mathrm{xCH}_{3}, \mathrm{~J} 6.6 \mathrm{~Hz}\right), 3.43(\mathrm{~m}$, $1 \mathrm{H}), 6.62(\mathrm{~s}, 1 \mathrm{H}, \mathrm{NH}$ bzd $), 6.77-6.82\left(\mathrm{~m}, 2 \mathrm{H}_{\text {arom }}\right), 6.95-6.99\left(\mathrm{~d}, 1 \mathrm{H}, \mathrm{H}_{\text {olefinic }} J 14.1 \mathrm{~Hz}\right), 6.98-7.00\left(\mathrm{~d}, 1 \mathrm{H}_{\text {arom }}, J 7.5 \mathrm{~Hz}\right)$, 6.99-7.43 ( $\left.\mathrm{m}, 5 \mathrm{H}_{\text {arom }}\right), 8.75$ (m, $1 \mathrm{H}, \mathrm{NH}_{\text {enaminone) }}, 15.12$, (bd-s, $\left.1 \mathrm{H}, \mathrm{OH}\right) .{ }^{13} \mathrm{C} \mathrm{NMR}\left(75.47 \mathrm{MHz}, \mathrm{CDCl}_{3}\right) \delta_{\mathrm{c}}: 23.5$, 50.5, 94.7, 117.5, 118.3, 120.7, 121.5, 124.5, 126.7, 127.5, 130.7, 132.7, 133.1, 136.9, 155.0, 163.2, 168.1, 178.3; IR (ATR) $v_{\max } / \mathrm{cm}^{-1}$ : 3254-3192 (NH), $3045(\mathrm{OH}) 1646$ (C=O), 1561 (C=N); HRMS (ESI-TOF) for $\mathrm{C}_{19} \mathrm{H}_{20} \mathrm{~N}_{3} \mathrm{O}_{2}$ $[\mathrm{M}+\mathrm{H}]^{+}$: calcd 322.1480 found 322.1461 .

(3Z)-3-((butylamino)methylene)-4-(2-hydroxyphenyl)-1,3-dihydro-2H-1,5-benzodiazepin-2-one (3d)

Yellow solid, yield 90\%, mp: 193-195 ${ }^{\circ} \mathrm{C} ;{ }^{1} \mathrm{H}$ NMR $\left(300 \mathrm{MHz}, \mathrm{CDCl}_{3}\right) \delta_{\mathrm{H}}: 0.91\left(\mathrm{t}, 3 \mathrm{H}, \mathrm{CH}_{3}, J 7.5 \mathrm{~Hz}\right), 1.34(\mathrm{~m}, 2 \mathrm{H}$, $\left.\mathrm{CH}_{2}\right), 1.54\left(\mathrm{~m}, 2 \mathrm{H}, \mathrm{CH}_{2}\right), 3.19$ (qd, $2 \mathrm{H}, \mathrm{CH}_{2}, 3^{3} J_{1} 8 \mathrm{~Hz}, 3_{2}^{3} 6 \mathrm{~Hz}$ ), 6.57 (s, $\left.1 \mathrm{H}, \mathrm{NHbzd}\right), 6.77-6.85\left(\mathrm{~m}, 2 \mathrm{H}_{\text {arom }}\right), 6.91$ (d, $\left.1 \mathrm{H}, \mathrm{H}_{\text {olefinic, }} J 13.8 \mathrm{~Hz}\right), 6.98-7.44\left(\mathrm{~m}, 6 \mathrm{H}_{\text {arom }}\right), 8.8(\mathrm{~m}, 1 \mathrm{H}, \mathrm{NH}$ enaminone), 15.2 (bd-s, $1 \mathrm{H}, \mathrm{OH}) .{ }^{13} \mathrm{C} \mathrm{NMR}(75.47 \mathrm{MHz}$, $\left.\mathrm{CDCl}_{3}\right) \delta_{\mathrm{C}}: 13.5,19.6,32.8,49.1,95.1,117.5,118.1,120.7,121.6,124.5,126.7,127.6,130.7,132.7,133.1$, 137.1, 157.1, 163.2, 167.9, 178.4; IR (ATR) $v_{\max } / \mathrm{cm}^{-1}: 3283-3179(\mathrm{NH}), 3056(\mathrm{OH}) 1641$ (C=O), 1578 (C=N); Crystal suitable for single-crystal $X$-ray diffraction analysis were grown from a mixture of $\mathrm{CH}_{2} \mathrm{Cl}_{2} / \mathrm{n}$-hexane solution (3:1 v/v) upon standing at ambient temperature. Crystal data for $3 \mathbf{d ~ C}_{20} \mathrm{H}_{21} \mathrm{~N}_{3} \mathrm{O}_{2}: \mathrm{Mr}=335.39$, monoclinic, $C 2 / c^{\prime}, a=26.0905(14), b=9.0775(4), c=15.1426(7) \AA ̊ \AA, \beta^{\circ}=97.260(3), V=3557.6(3) \AA^{3}, Z=8, \delta_{\text {calcd }}$ $=1.249 \mathrm{~g} / \mathrm{cm}^{3} ; \mathrm{F}(000) 1416 ; 3979$ reflections collected, 1937 independent reflections. Final agreement factors: $\mathrm{R}_{1}=0.1456$ (all observed) and 0.1418 with $\mathrm{I}>2 \mathrm{~s}(\mathrm{I}), \mathrm{wR}_{2}=0.0632$ (all observed) and 0.1144 with $\mathrm{I}>2 \mathrm{r}(\mathrm{I})$. GOF = 1.012. CCDC 1035304.HRMS (ESI-TOF) for $\mathrm{C}_{20} \mathrm{H}_{22} \mathrm{~N}_{3} \mathrm{O}_{2}[\mathrm{M}+\mathrm{H}]+$ : calcd, 336.1634 found 336.1610 .

(3Z)-4-(2-hydroxyphenyl)-3-((2,4,4-trimethylpentan-2-yl-amino)methylene)-1,3-dihydro-2H-1,5-benzodiaze-pin-2-one (3e). Yellow solid, yield 80\%, mp 195-198 ${ }^{\circ} \mathrm{C}$; ${ }^{1} \mathrm{H}$ NMR $\left(300 \mathrm{MHz}, \mathrm{CDCl}_{3}\right) \delta_{\mathrm{H}}: 0.95\left(\mathrm{~s}, 9 \mathrm{H}, 3 \times \mathrm{CH}_{3}\right), 1.20$ $\left(\mathrm{s}, 6 \mathrm{H}, 2 \mathrm{CH}_{3}\right), 1.46\left(\mathrm{~s}, 2 \mathrm{H}, \mathrm{CH}_{2}\right), 6.55(\mathrm{~s}, 1 \mathrm{H}, \mathrm{NHbzd}), 6.76-6.83\left(\mathrm{~m}, 2 \mathrm{H}_{\text {arom }}\right), 6.76-6.83\left(\mathrm{~d}, \mathrm{H}_{\text {arom }}, \mathrm{J} 8.1 \mathrm{~Hz}\right) 7.03-$

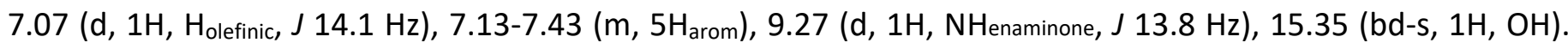
${ }^{13} \mathrm{C}$ NMR $\left(75.47 \mathrm{MHz}, \mathrm{CDCl}_{3}\right) \delta_{c}: 7.7,36.5,38.1,61.9,63.2,94.7,117.3,118.2,120.6,121.6,124.4,126.7$, 
127.5, 130.7, 132.7, 133.3, 137.1, 152.9, 163.3, 168.3, 178.3; IR (ATR) $v_{\max } / \mathrm{cm}^{-1}: 3224-3202(\mathrm{NH}), 3056(\mathrm{OH})$ $1644(\mathrm{C}=\mathrm{O}), 1557$ ( $\mathrm{C}=\mathrm{N}$ ); HRMS (ESI-TOF) for $\mathrm{C}_{24} \mathrm{H}_{29} \mathrm{~N}_{3} \mathrm{O}_{2}[\mathrm{M}+\mathrm{H}]+$ : calcd, 391.2260 found 391.2254.

(3Z)-3-((tert-butylamino)methylene)-4-(2-hydroxyphenyl)-1,3-dihydro-2H-1,5-benzodiazepin-2-one (3f) Orange solid, yield 77\%, mp 189-192 ${ }^{\circ} \mathrm{C}$; ${ }^{1} \mathrm{H}$ NMR $\left(300 \mathrm{MHz}, \mathrm{CDCl}_{3}\right) \delta_{\mathrm{H}}: 1.14\left(\mathrm{~s}, 9 \mathrm{H}, 3 \times \mathrm{CH}_{3}\right), 6.77-6.83(\mathrm{~m}$, $\left.2 \mathrm{H}_{\text {arom }}\right), 6.85\left(\mathrm{~s}, 1 \mathrm{H}, \mathrm{NH}_{\text {bzd }}\right), 6.99$ (d, $\left.1 \mathrm{H}_{\text {arom }}, J 8.1 \mathrm{~Hz}\right), 7.06\left(\mathrm{~d}, 1 \mathrm{H}, \mathrm{H}_{\text {olefinic, }} J 14.4 \mathrm{~Hz}\right), 7.08-7.44\left(\mathrm{~m}, 5 \mathrm{H}_{\text {arom }}\right), 9.05$ (d, $1 \mathrm{H}$, NHenaminone, J $14.1 \mathrm{~Hz}), 15.45(\mathrm{bd}-\mathrm{s}, 1 \mathrm{H}, \mathrm{OH}) .{ }^{13} \mathrm{C}$ NMR $\left(75.47 \mathrm{MHz}, \mathrm{CDCl}_{3}\right) \delta_{\mathrm{C}}: 16.1,43.8,94.9,117.6$, 118.2, 120.7, 121.6, 124.5, 126.8, 127.7, 130.8, 131.9, 133.5, 137.0, 153.7, 163.3, 168.8, 178.0; IR (ATR) $v_{\max } / \mathrm{cm}^{-1}$ : 3287-3147 (NH), $3060(\mathrm{OH}) 1650(\mathrm{C}=\mathrm{O}), 1567(\mathrm{C}=\mathrm{N})$; HRMS (ESI-TOF) for $\mathrm{C}_{20} \mathrm{H}_{22} \mathrm{~N}_{3} \mathrm{O}_{2}[\mathrm{M}+\mathrm{H}]+$ : calcd 336.1634 found 336.1625 .

(3Z)-4-(2-hydroxyphenyl)-3-((isobutylamino)methylene)-1,3-dihydro-2H-1,5-benzodiazepin-2-one

(3g).

Orange solid, yield $85 \%$; mp 160-163 ${ }^{\circ} \mathrm{C} ;{ }^{1} \mathrm{H}$ NMR $\left(300 \mathrm{MHz}, \mathrm{CDCl}_{3}\right) \delta_{\mathrm{H}}: 0.93\left(\mathrm{~d}, 6 \mathrm{H}, 2 \mathrm{xCH}_{3}, J 6.6 \mathrm{~Hz}\right), 1.78(\mathrm{~m}$, $1 \mathrm{H}), 3.72\left(\mathrm{t}, 2 \mathrm{H}, \mathrm{CH}_{2}, J 6.3 \mathrm{~Hz}\right), 6.72\left(\mathrm{~s}, 1 \mathrm{H}, \mathrm{NH}_{\mathrm{bzd}}\right), 6.76-6.85\left(\mathrm{~m}, 2 \mathrm{H}_{\text {arom }}\right), 6.89\left(\mathrm{~d}, 1 \mathrm{H}, \mathrm{H}_{\text {olefinic, }} J 13.8 \mathrm{~Hz}\right), 6.99-$ 7.45 (m, 6H arom), 8.77 (m, $1 \mathrm{H}, \mathrm{NH}$ enaminone]), 15.27, (bd-s, $1 \mathrm{H}, \mathrm{OH}) .{ }^{13} \mathrm{C} N M R\left(75.47 \mathrm{MHz}, \mathrm{CDCl}_{3}\right) \delta_{\mathrm{c}}: 19.7,29.7$, 57.1, 94.9, 117.5, 118.1, 120.8, 121.5, 124.4, 126.7, 127.1, 130.8, 132.7, 133.2, 136.9, 157.4, 163.1, 167.9, 178.6; IR (ATR) $v_{\max } / \mathrm{cm}^{-1}$ : 3289-3147 (NH), 3064 (OH) 1652 (C=O), 1567 (C=N); HRMS (ESI-TOF) for $\mathrm{C}_{20} \mathrm{H}_{22} \mathrm{~N}_{3} \mathrm{O}_{2}$ $[\mathrm{M}+\mathrm{H}]+$ : calcd, 336.1634 found 336.1623 .

(3Z)-3-((sec-butylamino)methylene)-4-(2-hydroxyphenyl)-1,3-dihydro-2H-1,5-benzodiazepin-2-one

(3h). Orange solid, yield $85 \%$; mp 156-158 ${ }^{\circ} \mathrm{C} ;{ }^{1} \mathrm{H} \mathrm{NMR}\left(300 \mathrm{MHz}, \mathrm{CDCl}_{3}\right) \delta_{\mathrm{H}}: 0.94\left(\mathrm{t}, 3 \mathrm{H}, \mathrm{CH}_{3}, J 7.2 \mathrm{~Hz}\right), 1.21(\mathrm{~d}, 3 \mathrm{H}$, $\left.\mathrm{CH}_{3}, J 6.6 \mathrm{~Hz}\right), 1.54\left(\mathrm{~m}, 2 \mathrm{H}, \mathrm{CH}_{2}\right), 3.14(\mathrm{~m}, 1 \mathrm{H}, \mathrm{CH}), 6.71(\mathrm{~s}, 1 \mathrm{H}, \mathrm{NH}$ zzd $), 6.74-6.80\left(\mathrm{~m}, 2 \mathrm{H}_{\text {arom }}\right), 6.96\left(\mathrm{~d}, 1 \mathrm{H}, \mathrm{H}_{\text {olefinic }}\right.$, J 13.8 Hz), 6.97-7.00 (d, $\left.1 \mathrm{H}_{\text {arom }}, J 8.4 \mathrm{~Hz}\right), 7.02-7.45\left(\mathrm{~m}, 5 \mathrm{H}_{\text {arom }}\right), 8.76(\mathrm{~m}, 1 \mathrm{H}, \mathrm{NH}$ enaminone $), 15.00,(\mathrm{bd}-\mathrm{s}, 1 \mathrm{H}, \mathrm{OH})$. ${ }^{13} \mathrm{C}$ NMR $\left(75.47 \mathrm{MHz}, \mathrm{CDCl}_{3}\right) \delta_{c}: 9.8,20.7,29.9,56.2,94.4,117.7,118.7,120.2,120.3,123.9,126.2,127.0$, $130.2,132.1,132.7,136.6,155.0,162.8,167.6,177.9 ;$ IR (ATR) $v_{\max } / \mathrm{cm}^{-1}: 3286-3168$ (NH), 3043 (OH) 1640 (C=O), 1576 (C=N); HRMS (ESI-TOF) for $\mathrm{C}_{20} \mathrm{H}_{22} \mathrm{~N}_{3} \mathrm{O}_{2}[\mathrm{M}+\mathrm{H}]+$ : calcd, 336.1636 found 335.1621.

(3Z)-3-((hexylamino)methylene)-4-(2-hydroxyphenyl)-1,3-dihydro-2H-1,5-benzodiazepin-2-one (3i). Yellow solid, yield 65\%, m.p 198-195 ${ }^{\circ} \mathrm{C}$; ${ }^{1} \mathrm{H}$ NMR $\left(300 \mathrm{MHz}, \mathrm{CDCl}_{3}\right) \delta_{\mathrm{H}}: 0.90\left(\mathrm{t}, 3 \mathrm{H}, \mathrm{CH}_{3}, J 6.6 \mathrm{~Hz}\right), 1.31\left(\mathrm{~m}, 6 \mathrm{H}, 3 \times \mathrm{CH}_{2}\right)$, $1.57\left(\mathrm{~m}, 2 \mathrm{H}, \mathrm{CH}_{2}\right), 3.20\left(\mathrm{qd}, 2 \mathrm{H}, \mathrm{CH}_{2},{ }^{3} \mathrm{~J}_{1}={ }^{3} \mathrm{~J}_{2} 6.6 \mathrm{~Hz}\right.$ ), $6.69\left(\mathrm{~s}, 1 \mathrm{H}, \mathrm{NH}_{\mathrm{bzd}}\right), 6.79-6.88\left(\mathrm{~m}, 2 \mathrm{H}_{\text {arom }}\right), 6.93(\mathrm{~d}, 1 \mathrm{H}$, $\mathrm{H}_{\text {olefinic }}, \mathrm{J} 13.8 \mathrm{~Hz}$ ), 7.01-7.46 (m, 6Harom), 8.85 (m, $1 \mathrm{H}, \mathrm{NH}$ enaminone), 15.2 (bd-s, $\left.1 \mathrm{H}, \mathrm{OH}\right) .{ }^{13} \mathrm{C} \mathrm{NMR}(75.47 \mathrm{MHz}$, $\left.\mathrm{CDCl}_{3}\right) \delta_{\mathrm{c}}: 13.4,21.9,25.6,30.8,31.3,49.4,94.5,117.5,118.3,120.7,121.5,124.5,126.8,127.5,130.8,132.3$, 132.8, 137.1, 156.5, 162.8, 167.5, 177.8; IR (ATR) $v_{\max } / \mathrm{cm}^{-1}: 3302-3191$ (NH), 3057 (OH) 1648 (C=O), 1580 (C=N); HRMS (ESI-TOF) for $\mathrm{C}_{22} \mathrm{H}_{26} \mathrm{~N}_{3} \mathrm{O}_{2}[\mathrm{M}+\mathrm{H}]+$ : calcd, 364.1947 found 364.1940.

(3Z)-3-((cyclohexylamino)methylene)-4-(2-hydroxyphenyl)-1,3-dihydro-2H-1,5-benzodiazepin-2-one

(3j).

Yellow solid, yield 84\%, m.p 191-194 ${ }^{\circ} \mathrm{C} ;{ }^{1} \mathrm{H}$ NMR $\left(300 \mathrm{MHz}, \mathrm{CDCl}_{3}\right) \delta_{\mathrm{H}}: 1.019-1.91(\mathrm{~m}, 10 \mathrm{H}$-cyclohexyl), $3.03(\mathrm{~m}$, $1 \mathrm{H}$-cyclohexyl) $6.58(\mathrm{~s}, 1 \mathrm{H}, \mathrm{NH}$ bzd $), 6.77-6.85\left(\mathrm{~m}, 2 \mathrm{H}_{\text {arom }}\right), 6.99\left(\mathrm{~d}, 1 \mathrm{H}, \mathrm{H}_{\text {olefinic, }} J 13.8 \mathrm{~Hz}\right), 7.00-7.43\left(\mathrm{~m}, 6 \mathrm{H}_{\text {arom }}\right.$ ), $8.82\left(\mathrm{dd}, 1 \mathrm{H}, \mathrm{NH}\right.$ enaminone, $\left.J_{1} 5.7 \mathrm{~Hz}, J_{2} 11.4 \mathrm{~Hz}\right), 15.41(\mathrm{bd}-\mathrm{s}, 1 \mathrm{H}, \mathrm{OH}) .{ }^{13} \mathrm{C} \mathrm{NMR}\left(75.47 \mathrm{MHz}, \mathrm{CDCl}_{3}\right) \delta_{\mathrm{c}}: 24.5,25.0$, 33.8, 57.5, 94.7, 117.5, 118.1, 120.7, 121.5, 124.5, 126.7, 127.5, 130.7, 132.7, 133.1, 136.9, 155.1, 163.2, 168.1, 178.3; IR (ATR) $v_{\max } / \mathrm{cm}^{-1}: 3317-3197$ (NH), 3072 (OH) 1649 (C=O), 1588 (C=N); HRMS (ESI-TOF) for $\mathrm{C}_{22} \mathrm{H}_{24} \mathrm{~N}_{3} \mathrm{O}_{2}[\mathrm{M}+\mathrm{H}]+$ : calcd, 362.1790 found 362.1780 .

(3Z)-4-(2-hydroxyphenyl)-3-((phenylamino)methylene)-1,3-dihydro-2H-1,5-benzodiazepin-2-one (3k). Yellow solid 55\%; mp 232-235 ${ }^{\circ} \mathrm{C}$; ${ }^{1} \mathrm{H}$ NMR $\left(300 \mathrm{MHz}, \mathrm{CDCl}_{3}\right) \delta_{\mathrm{H}}: 6.70-6.90\left(\mathrm{~m}, 1 \mathrm{H}_{\text {arom }}\right), 6.76\left(\mathrm{~s}, 1 \mathrm{H}, \mathrm{NH}_{\mathrm{bzd}}\right), 6.96-7.29$ $\left(\mathrm{m}, 11 \mathrm{H}_{\text {arom }}\right), 7.34\left(\mathrm{~d}, 1 \mathrm{H}, \mathrm{H}_{\text {olefinic, }} J 13.2 \mathrm{~Hz}\right), 7.50\left(\mathrm{~m}, 1 \mathrm{H}_{\text {arom }}\right), 10.17(\mathrm{~d}, 1 \mathrm{H}, \mathrm{NH}$ enaminone, J $13.2 \mathrm{~Hz}), 15.07$ (bd-s, $1 \mathrm{H}, \mathrm{OH}) .{ }^{13} \mathrm{C}$ NMR $\left(75.47 \mathrm{MHz}, \mathrm{CDCl}_{3}\right) \delta_{\mathrm{C}}: 98.8,116.1,117.6,117.8,121.0,123.8,124.4,126.6,127.4,129.3$, $130.3,131.5,132.7,136.3,138.9,146.6,156.8,162.6,166.3,176.8$; IR (ATR) $v_{\max } / \mathrm{cm}^{-1}: 3240-3184$ (NH), 3107 (OH) 1651 (C=O), 1576 (C=N); HRMS (ESI-TOF) $\mathrm{C}_{22} \mathrm{H}_{18} \mathrm{~N}_{3} \mathrm{O}_{2}[\mathrm{M}+\mathrm{H}]+$ : calcd, 356.1321 found 356.1317. 
(3Z)-4-(2-hydroxyphenyl)-3-((4-methoxyphenylamino)methylene)-1,3-dihydro-2H-1,5-benzodiazepin-2-one (31). Yellow solid ${ }_{2}$ yield $60 \%, \mathrm{mp} 200-198{ }^{\circ} \mathrm{C} ;{ }^{1} \mathrm{H} \mathrm{NMR}\left(300 \mathrm{MHz}, \mathrm{CDCl}_{3}\right) \delta_{\mathrm{H}}: 3.68\left(\mathrm{~s}, 3 \mathrm{H}, \mathrm{CH}_{3}\right), 6.70-6.82(\mathrm{~m}$, $\left.4 \mathrm{H}_{\text {arom }}\right), 6.90$ (s, $1 \mathrm{H}, \mathrm{NH}$ bzd $), 6.94-7.09$ (m, 3H $\left.\mathrm{H}_{\text {arom }}\right), 7.20$ (d, $\left.1 \mathrm{H}, \mathrm{H}_{\text {olefinic, }} J 12.0 \mathrm{~Hz}\right), 7.24-7.47$ (m, $\left.5 \mathrm{H}_{\text {arom }}\right), 10.24$ (d, $1 \mathrm{H}$, NHenaminone, J= $12.9 \mathrm{~Hz}$ ), $14.93(\mathrm{bd}-\mathrm{s}, 1 \mathrm{H}, \mathrm{OH}) .{ }^{13} \mathrm{C} N M R\left(75.47 \mathrm{MHz}, \mathrm{CDCl}_{3}\right) \delta_{\mathrm{c}}: 55.5,98.1,115.0,118.0$, 118.2, 118.3, 120.8, 121.2, 124.8, 127.0, 127.8, 130.7, 132.3, 133.0, 133.1, 137.1, 148.38, 158.2, 163.0, 167.1, 178.0; IR (ATR) $v_{\max } / \mathrm{cm}^{-1}: 3237-3212(\mathrm{NH}), 3101(\mathrm{OH}) 1654$ (C=O), 1591 (C=N); HRMS (ESI-TOF) $\mathrm{C}_{23} \mathrm{H}_{20} \mathrm{~N}_{3} \mathrm{O}_{3}[\mathrm{M}$ $+\mathrm{H}]+$ : calcd 386.1426 found 386.1435 .

(3Z)-3-((4-chlorophenylamino)methylene)-4-(2-hydroxyphenyl)-1,3-dihydro-2H-1,5-benzodiazepin-2-one (3m). Yellow solid ${ }_{2}$ yield 50\%, mp: $220-224{ }^{\circ} \mathrm{C} ;{ }^{1} \mathrm{H}$ NMR $\left(300 \mathrm{MHz}, \mathrm{CDCl}_{3}\right) \delta_{\mathrm{H}}: 6.87-6.91\left(\mathrm{~d}, 1 \mathrm{H}, \mathrm{H}_{\text {olefinic, }} J 12.0\right.$ $\mathrm{Hz}), 6.86-6.92\left(\mathrm{~m}, 1 \mathrm{H}_{\text {arom }}\right), 7.04\left(\mathrm{~s}, 1 \mathrm{H}, \mathrm{NH}_{\mathrm{bzd}}\right), 7.07-7.58\left(\mathrm{~m}, 11 \mathrm{H}_{\text {arom }}\right), 10.20(\mathrm{~d}, 1 \mathrm{H}$, NHenaminone, J $12.1 \mathrm{~Hz}), 15.00$ (bd-s, $1 \mathrm{H}, \mathrm{OH}) .{ }^{13} \mathrm{C}$ NMR $\left(75.47 \mathrm{MHz}, \mathrm{CDCl}_{3}\right) \delta_{\mathrm{c}}: 99.1,115.1,117.4,118.2,118.3,120.7,121.2,124.3,127.0$, 127.9, 130.7, 132.1, 133.0, 133.9, 137.1, 146.2, 156.1, 162.2, 166.1, 176.0; IR (ATR) $v_{\max } / \mathrm{cm}^{-1}: 3207-3154$ (NH), $3047(\mathrm{OH}) 1654(\mathrm{C}=\mathrm{O}), 1566$ (C=N); HRMS (ESI-TOF) $\mathrm{C}_{22} \mathrm{H}_{17} \mathrm{CIN}_{3} \mathrm{O}_{2}[\mathrm{M}+\mathrm{H}]+$ : calcd, 390.0931 found 390.0927.

Single-crystal X-ray diffraction analysis. A yellow prism of crystals of $\mathbf{3 d}$, was chosen by size, habit, and polarized light microscopy and mounted on a glass fiber. Intensity data were collected at ambient temperature on an Enraf-Nonius CAD-4 diffractometer equipped with graphite monochromated Mo Ka radiation $\left(\lambda_{\text {MoK }_{\alpha}}=\right.$ $0.71073 \AA$ A). Reflection data were corrected for Lorentz-polarization effects but not for absorption. The structure was solved by direct methods and subsequent difference Fourier techniques (SIR-92) ${ }^{23}$ and refined with SHELXL-2013. ${ }^{24}$ Non-hydrogen atoms were refined with anisotropic thermal parameters. Hydrogen atoms were placed in idealized positions on parent atoms in the final refinement.

Further crystallographic data can be obtained free of charge on application to CCDC, 12 Union Road, Cambridge CB2 1EZ, UK (fax: (+44)1223-336-033; e-mail: deposit@ccdc.cam.ac.uk, website http://www.ccdc.cam.ac.uk).CCDC 1035304.

\section{Antibacterial test of cis- $\beta$-enaminone-1,5-benzodiazepines $3(\mathrm{a}-\mathrm{m})$}

The antibacterial activity of compounds $3 a-m$ was tested against different microorganisms, including reference strains consisting of Gram-negative rods: Escherichia coli (ATCC35218), Pseudomonas aeruginosa (ATCC 27853), Salmonella typhimurium (LT2 DT104), Listeria monocytogenes (ATCC 19115), and Gram-positive cocci: Staphylococcus aureus (ATCC 25923) and Enterococcus faecalis (ATCC 29212), Micrococcus luteus (NCIMB8166), Bacillus cereus (ATCC11778), and Staphylococcus epidermidis (CIP106510). Strains were cultured over night at $37^{\circ} \mathrm{C}$ in Muller Hinton agar. The minimal inhibition concentration (MIC) was determined by micro-titer plate dilution method using sterile $10 \% \mathrm{H}_{2} \mathrm{O}$ solution to dissolve samples. ${ }^{25}$ Streptomycin obtained from commercial sources was used as reference antibacterial agent.

\section{Acknowledgements}

The authors acknowledge Dr Basma Hamdi (Faculty of Sciences of Sfax) for the X-ray crystallographic analysis, and Miss Amna Benzarti, NMR service at the Faculty of Monastir, University of Monastir for the 1D and 2D NMR analysis and to the Ministry of Higher Education and Scientific Research of Tunisia for financial support (LR11ES39). 


\section{References}

1. Evans, B. E.; Rittle, K. E.; Bock, M. G.; DiPardo, R. M.; Freidinger, R. M.; Whitter, W. L.; Lundell, G. F.; Veber, D. F.; Anderson, P. S.; Chang, R. S.; Lotti, V. J.; Cerino, D. J.; Chen, T. B.; Kling, P. J.; Kunkel, K. A.; Springer, J. P.; Hirshfield, J. J. Med. Chem. 1988, 31, 2235 - 2246.

https://doi.org/10.1021/jm00120a002

2. Chen, X.; Zheng, Y.; Shu, C.; Yuan, W.; Liu, B.; Zhang, X. J. Org. Chem. 2011, 76, 9109-9115. https://doi.org/10.1021/jo201334n

3. Navjeet, K. Int. J Pharm Bio Sci. 2013, 4(2), 318-337.

4. Rajesh, B. Patil.; Sanjay, D. Sawant.; Kavya, V. Reddy.; Milind, Shirsat. Res. J. Pharm., Biol. Chem. Sci. 2015, 6 (2), 381-391.

5. Ponnuswamy, S.; Akila, A.; Deepa Rajakumari, D.; Shreevidhyaa Suressh, V.; Usha, G. J. Chem. Sci. 2015, 127 (11), 2051-2061. https://doi.org/10.1007/s12039-015-0974-z

6. Kamal, A.; Shankaraiah, N.; Prabhakar, S.; Reddy, C.R.; Markandeya, N.; Laxma, K.; Devaiah, X.; Bioorg. Med. Chem. Lett. 2008, 18, 2434-2439.

https://doi.org/10.1016/j.bmcl.2008.02.047

7. Claramunt, Rosa M.; Alkorta, Ibon.; Elguero, José. Comput. Theor. Chem. 2013, 1019, 108-115. https://doi.org/10.1016/i.comptc.2013.07.002

8. Fitos, I.; Visy, J.; Zsila, F.; Mády, G.; Simonyi, M. Bioorg. Med.Chem. 2007, 15, 4857-4862. https://doi.org/10.1016/j.bmc.2007.04.060

9. Greenhill, J. V. Chem. Soc. Rev. 1977, 6, 277-294. http://dx.doi.org/10.1039/CS9770600277

10. Edafiogho, I. O.; Phillips, O. A.; Udo, E. E.; Samuel, Sa.; Rethish, B. Eur J Med. Chem. 2009, 44, 967-975. https://doi.org/10.1016/i.ejmech.2008.07.005

11. Xu, X.; Du, P.; Cheng, D.; Wang, H.; Li, X. Chem. Commun. 2012, 48, 1811-1813. http://dx.doi.org/10.1039/C2CC16997E

12. Fader L. D.; Bethell, R.; Bonneau, P.; Bös, M.; Bousquet, Y.; Cordingley, M.G.; Coulombe, R.; Deroy, P.; Faucher, AM.; Gagnon, A.; Goudreau, N.; Grand-Maître C, Guse, I.; Hucke O.; Kawai S. H.; Lacoste, JE.; Landry, S.; Lemke, C. T.; Malenfant, E.; Mason, S.; Morin S.; O’Meara, J.; Simoneau, B.; Titolo, S.; Yoakim, C. Bioorg. Med. Chem. Lett. 2011, 21. 398-404.

https://doi.org/10.1016/j.bmcl.2010.10.131

13. Hamdi, N.; Lidrissi, C.; Saoud, M.; Romerosa Nievas, A.; Zarrouk,H. Chem. Heterocycl. Compd. 2006, 42 (3), 320-325.

https://doi.org/10.1002/jhet.5570310242

14. Khodairy, A. Synthetic Communications. 2011, 41, 612-621. https://doi.org/10.1080/00397911003629507

15. Gharbi, R.; Trimèche, B.; Martin, M.T.; Mighri, Z. Heterocycl. Commun. 2002, 8 (4), 335-338.

16. Trimèche, B.; Gharbi, R.; El Houla, S.; Martin, M.T.; Nuzillard J.M.; Mighri, Z. J. Chem. Res. 2004, 5, 170173. https://doi.org/10.3184/0308234041640636.

17. Gharbi, R.; Ben-Youssef, M.; Martin, M.T.; Mighri, Z. J. Chem. Res. 2005, 257-261. https://doi.org/10.1002/jhet.5570420128 
18. Nsira. A.; Gharbi, R.; Msaddek, M. CR Chimie. 2014, 17 (12), 1242-1249. https://doi.org/10.1016/j.crci.2014.01.007

19. Escobar, C. A.; Donoso-Tauda, O.; Araya-Maturana, R.; Vega, A. 2007, Acta Cryst. Section C63 o4260430.

https://doi.org/10.1107/S0108270107024420

20. Zhuo, J.-C. Magn. Reson. Chem. 1998, 36, 565-572.

https://doi.org/10.1002/(SICI)1097-458X(199808)36:8<565::AID-OMR338>3.0.CO;2-H

21. Mtiraoui, H.; Gharbi, R.; Msaddek, M.; Bretonnière, Y.; Andraud, C.; Sabot, C.; Renard, PY. J. Org. Chem. 2016, 81, 4720-4727.

https://doi.org/10.1021/acs.joc.6b00627

22. Karplus, Martin. J. Am. Chem. Soc. 1963, 85 (18), 2870-2871.

23. Altomare, A.; Burla, M.C.; Camalli, G.; Cascarano, G.; Giacovazzo, C.; Gualiardi, A.; G. Polidori, J. (IUCr) J. App. Cryst. 1994, 27, 435.

https://doi.org/10.1107/S002188989400021X

24. Sheldrick, G.M. Acta Crystallogr. 2008, A64, 112-122.

https://doi.org/10.1107/S0108767307043930

25. Eloff, J. N., Planta Med. 1998, 64, 711-713.

https://doi.org/10.1055/s-2006-957563 